\title{
ARTICLE
}

Chronic lymphocytic leukemia

\section{COVID-19 severity and mortality in patients with chronic lymphocytic leukemia: a joint study by ERIC, the European Research Initiative on CLL, and CLL Campus}

\author{
Lydia Scarfò $^{1} \cdot$ Thomas Chatzikonstantinou $^{2} \cdot$ Gian Matteo Rigolin $^{3} \cdot$ Giulia Quaresmini $^{4} \cdot$ Marina Motta $^{5}$ •
} Candida Vitale ${ }^{6} \cdot$ Jose Antonio Garcia-Marco $\mathbb{D}^{7} \cdot$ José Ángel Hernández-Rivas $\mathbb{D}^{8} \cdot$ Fatima Mirás ${ }^{9} \cdot$ Mónica Baile $^{10}$.

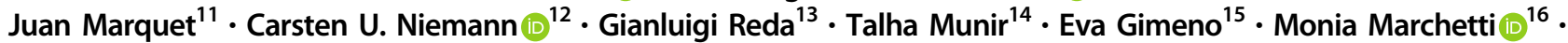
Francesca Maria Quaglia ${ }^{17}$. Marzia Varettoni ${ }^{18}$. Julio Delgado ${ }^{19}$ - Sunil lyengar ${ }^{20} \cdot$ Ann Janssens $^{21} \cdot$ Roberto Marasca $^{22}$. Angela Ferrari ${ }^{23}$. Carolina Cuéllar-García $\mathbb{B D}^{24} \cdot$ Gilad Itchaki $^{25} \cdot$ Martin Špaček $^{26} \cdot$ Lorenzo De Paoli $^{27} \cdot$ Luca Laurenti $^{28}$. Mark-David Levin ${ }^{29}$. Enrico Lista ${ }^{30}$. Francesca R. Mauro ${ }^{31}$ - Martin Šimkovič ${ }^{32}$ • Ellen Van Der Spek ${ }^{33}$. Elisabeth Vandenberghe $\mathrm{e}^{34} \cdot$ Livio Trentin ${ }^{35} \cdot$ Ewa Wasik-Szczepanek $^{36} \cdot$ Rosa Ruchlemer $^{37} \cdot$ Dominique Bron $^{38}$. Maria Rosaria De Paolis ${ }^{39}$ - Giovanni Del Poeta ${ }^{40}$ - Lucia Farina ${ }^{41}$ - Myriam Foglietta ${ }^{42}$ - Massimo Gentile ${ }^{43}$. Yair Herishanu ${ }^{44}$. Tobias Herold ${ }^{45}$. Ozren Jaksic ${ }^{46}$. Arnon P. Kater ${ }^{47}$ - Sabina Kersting ${ }^{48} \cdot$ Lara Malerba $^{49}$. Lorella Orsucci $^{50}$ - Viola Maria Popov $\mathbb{D}^{51} \cdot$ Paolo Sportoletti $\mathbb{D}^{52} \cdot$ Mohamed Yassin $^{53} \cdot$ Barbara Pocali $^{54} \cdot$ Gabor Barna $^{55}$.

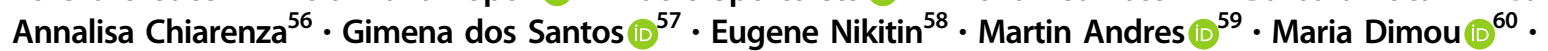
Michael Doubek $\mathbb{D}^{61}$ - Alicia Enrico ${ }^{62} \cdot$ Yervand Hakobyan ${ }^{63} \cdot$ Olga Kalashnikova $^{64}$ - Macarena Ortiz Pareja ${ }^{65}$. Maria Papaioannou ${ }^{66}$. Davide Rossi ${ }^{67,68}$. Nimish Shah ${ }^{69}$. Amit Shrestha ${ }^{70}$ - Oana Stanca ${ }^{71}$ - Niki Stavroyianni ${ }^{2}$. Vladimir Strugov $^{72}$ - Constantine Tam $^{73} \cdot$ Mihnea Zdrenghea $^{74} \cdot$ Marta Coscia $^{6} \cdot$ Kostas Stamatopoulos $^{75}$.

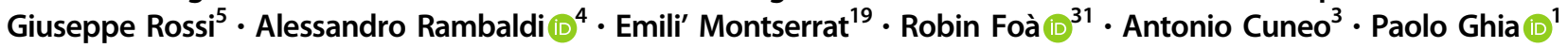

Received: 13 June 2020 / Revised: 21 June 2020 / Accepted: 30 June 2020 / Published online: 9 July 2020

(c) The Author(s), under exclusive licence to Springer Nature Limited 2020

\begin{abstract}
Chronic lymphocytic leukemia (CLL) is a disease of the elderly, characterized by immunodeficiency. Hence, patients with CLL might be considered more susceptible to severe complications from COVID-19. We undertook this retrospective international multicenter study to characterize the course of COVID-19 in patients with CLL and identify potential predictors of outcome. Of 190 patients with CLL and confirmed COVID-19 diagnosed between 28/03/2020 and 22/05/2020, 151 (79\%) presented with severe COVID-19 (need of oxygen and/or intensive care admission). Severe COVID-19 was associated with more advanced age ( $\geq 65$ years) (odds ratio 3.72 [95\% CI 1.79-7.71]). Only 60 patients (39.7\%) with severe COVID-19 were receiving or had recent ( $\leq 12$ months) treatment for CLL at the time of COVID-19 versus 30/39 (76.9\%) patients with mild disease. Hospitalization rate for severe COVID-19 was lower $(p<0.05)$ for patients on ibrutinib versus those on other regimens or off treatment. Of 151 patients with severe disease, 55 (36.4\%) succumbed versus only 1/38 (2.6\%) with mild disease; age and comorbidities did not impact on mortality. In CLL, (1) COVID-19 severity increases with age; (2) antileukemic treatment (particularly BTK inhibitors) appears to exert a protective effect; (3) age and comorbidities did not impact on mortality, alluding to a relevant role of CLL and immunodeficiency.
\end{abstract}

These authors contributed equally: Lydia Scarfò, Thomas Chatzikonstantinou, Gian Matteo Rigolin

These authors jointly supervised this work: Robin Foà, Antonio Cuneo, Paolo Ghia

Kostas Stamatopoulos

kostas.stamatopoulos@certh.gr

Supplementary information The online version of this article (https:// doi.org/10.1038/s41375-020-0959-x) contains supplementary material, which is available to authorized users.

\author{
$\triangle$ Paolo Ghia \\ ghia.paolo@hsr.it
}

Extended author information available on the last page of the article 


\section{Introduction}

Coronavirus disease 2019 (COVID-19), the new pandemic caused by coronavirus 2 (SARS-COV-2), has heavily affected the population worldwide since first recognized in December 2019 in Wuhan, China [1]. The disease follows a very heterogenous course, where the vast majority of patients are asymptomatic or experience few and mild symptoms, while others experience a very aggressive clinical course and progress rapidly to severe acute lung injury and acute respiratory distress syndrome, often associated with multiorgan failure (MOF) [2].

Evidence is gathering on the double-faceted role of the immune system in SARS-CoV-2 infection. An intact immune response is needed to face the initial infection and clear the virus, with patients recovering from the infection demonstrating evidence of humoral neutralizing antibodies [3]. On the other hand, the severity of COVID-19 and MOF occurrence seem to be related to clinical and laboratory parameters of inflammatory response (lymphopenia, hypoalbuminemia, higher levels of alanine aminotransferase, lactate dehydrogenase, C-reactive protein, ferritin, and D-dimer) and markedly higher levels of proinflammatory cytokines (interleukin-2 receptor, IL6, IL-10, and tumor necrosis factor$\alpha)$ [4]. On these grounds, therapies targeting inflammation (e.g., IL6/IL6R antibodies or steroids) have been used and shown potential benefit in full-blown COVID-19 patients [5].

Symptomatic disease and severe disease manifestations are more prevalent in elderly and co-morbid individuals [6]. The role of concomitant cancer, particularly for hematologic malignancies, remains unclear [7-10], with initial evidence suggesting an increased risk of infection and of experiencing a rapid disease course in patients with cancer [6]. Studies from China have reported a statistically higher incidence of severe events (including intensive care unit admission/ventilation/death) in infected individuals with cancer compared to those without. Moreover, in a recent series focused on patients with hematologic neoplasms hospitalized for COVID-19, those without active cancer (watch \& wait strategy and remission status) presented a favorable outcome [11]. In that cohort, six out of six patients diagnosed with chronic lymphocytic leukemia (CLL) survived the infection [11]. A similarly favorable outcome was reported in a Spanish cohort focusing on patients with CLL, where all four affected cases recovered from COVID-19 without intensive care admission [12]. Besides this report, only anecdotal CLL cases have been reported so far [13].

CLL is a paradigmatic example of malignancy associated with impaired immune responses to common pathogens in a context of a profound immune dysregulation reflected by hypogammaglobulinemia, qualitative and quantitative B- and $\mathrm{T}$-cell defects, including impaired response to vaccination, and $\mathrm{CD} 4^{+}$lymphopenia, as well innate immune dysfunction and neutropenia $[14,15]$. This can be further exacerbated by antileukemic treatments that by definition impact on the host immune system, some applied continuously until progression [16]. This could arguably compromise the capacity of patients with CLL to clear the virus, with a possible higher infection risk for this population. Conversely, immunodeficiency might as well be protective against severe COVID-19 and MOF, limiting excessive activation of the immune system. In this context, the role of novel targeted therapies such as Bruton tyrosine kinase (BTK) inhibitors, with overall antiinflammatory activity due to effects on both innate immune cells and platelets, has also been proposed as potentially beneficial against severe disease manifestations [17]. Nevertheless, patients with CLL tend to be elderly and carry several comorbidities, representing, in principle, prototypic patients who may suffer the most severe consequences of a SARSCoV-2 infection.

Limited data are currently available on the clinical course of COVID-19 patients with CLL and the potential impact of antileukemic treatment on the severity and outcome of COVID-19. Thus, we undertook this retrospective international multicenter study in order to characterize the course of COVID-19 in patients with CLL and identify potential predictors of outcome.

\section{Materials and methods}

\section{Data collection}

This is a retrospective international multicenter study of the European Research Initiative on CLL (ERIC) and the CLL Campus. Participating members received a survey during the COVID-19 pandemic, using a cloud-based online platform. Investigators who confirmed patients with CLL and COVID-19 in their patient cohort were asked to fill a template with relevant clinical information. The study was approved by the institutional ethics committee, informed consent was obtained from all subjects. Data collection took place between March 28 and May 22, 2020. The full list of participating countries is reported in Supplementary Table 1.

Data extracted from the medical records included: baseline demographics, date of CLL diagnosis, CLL treatment status, presence, number, and type of comorbidities, date of COVID-19, COVID-19 symptoms, COVID-19 management, treatment, and outcome. Of the 190 patients studied, four Spanish cases were previously published in extenso [12], 47 patients were included in a report describing the impact of SARS-CoV-2 pandemic infection on the practical management of CLL in Italy with only limited clinical data [18]. 
Overall survival (OS) was defined as the time from suspected COVID-19 to death or last follow-up date.

In keeping with international practice, patients were deemed to have COVID-19 if an RT-PCR assay test from a throat or nose swab was positive for SARS-CoV-2. Patients with only radiological or clinical suspicion of COVID-19 yet testing negative by RT-PCR were not further analyzed.

Establishment of CLL diagnosis, treatment decisions, review of medical history, and assessment of patient status were performed by the local teams following international guidelines [19].

Severe COVID-19 was defined as hospitalization and need of oxygen or admission into an intensive care unit; nonsevere/mild COVID-19 was defined as confinement at home or hospitalization without need of oxygen. Hospitalized patients were defined as those with severe COVID-19 and those requiring hospitalization without need of oxygen.

\section{Statistical analysis}

Subgroups analyses were performed, stratifying patients based on (1) clinico-biological characteristics (age, comorbidities, gender); (2) severity of COVID-19 (severe vs nonsevere); (3) antileukemic treatment (ongoing or previous last therapy within the last 12 months vs never treated or previous last therapy $>12$ months, considering that therapyrelated immunosuppresion after chemoimmunotherapy (CIT) may persist in the first year after discontinuation). Subgroup analyses of patients with severe COVID-19 were carried out to better identify risk in this cohort of patients: these analyses stratified between untreated vs treated patients; and, those on treatment or recently treated vs never treated or off treatment for $>12$ months.

Means between groups were compared using independent group $t$-test and the Mann-Whitney test for normally and abnormally distributed data, respectively. Proportions for categorical variables were compared by chi-square or Fisher's test when appropriate. Survival outcomes were analyzed according to the Kaplan-Meier estimator. The analysis was performed using IBM SPSS version 26.0, Microsoft Excel Office 365, and Graphpad Prism v8.4.2.

\section{Results}

\section{Survey results}

One-hundred and twenty-one investigators from 118 sites completed the survey; most participants were hematologists. Among 121 responders, 58 confirmed the presence of COVID-19 cases in their CLL patient cohort. Most responders were from Europe (74.6\%), followed by Asia (13.6\%), Latin America (4.2\%), Africa (3.4\%), Canada
(2.5\%), and Australia (1.7\%). At the time of launching the data collection, we became aware of ongoing US collaborative initiatives on the same topic; therefore, in order to avoid potential redundancy, US sites were intentionally excluded from our survey.

All sites participating in the survey except one $(0.8 \%)$ reported implementing preventive measures for patients with CLL regarding the SARS-CoV-2 pandemic, applied to all patients with CLL $(57.0 \%)$ or only those attending the clinic (43.0\%). In the vast majority of cases (105/118 sites) these measures were implemented according to national recommendations for the SARS-CoV-2 pandemic. The main actions taken included frequent hand washing and wearing masks and gloves for health care professionals, sanitizers at the entrance of the visiting room, postponing visits, and implementing remote visit platforms. The full list of measures is reported in Supplementary Table 2.

\section{COVID-19 cases: site and patient disposition}

Among 221 cases with suspected COVID-19 based on presenting symptoms and/or imaging findings, 206 underwent molecular testing (RT-PCR) on nasopharyngeal swabs for SARS-CoV-2; of these, 190 (92.2\%) tested positive by RT-PCR and were selected as the study cohort.

Information on the total number of patients followed at each site was available for 47 participating sites, contributing $82.1 \%$ of all COVID-19 patients with CLL in our series. Collectively, these sites reported 156 COVID-19 cases among 15,083 (1.0\%) patients with CLL attended at the respective institutions. The proportion of patients developing symptomatic COVID-19 requiring hospitalization was 141/15,083 (0.9\%).

Patient characteristics, including CLL and treatment status at the time of COVID-19, are shown in Table 1. Median age at COVID-19 diagnosis was 72 years (range 48-94), with the expected male: female ratio of 2.0. At the time of COVID-19, 76\% of patients (145/190) carried one or more comorbidities, with a median number of 2 (range 1-9): the most frequent concerned hypertension (78 cases, $54 \%$ ), cardiovascular diseases (42 cases, 29\%), diabetes mellitus (35 cases, 24\%), chronic obstructive pulmonary disease (COPD, 9 cases, 6\%), other respiratory diseases $(21,14 \%)$, other malignancies $(18,12 \%)$.

Regarding CLL history, 73 (38.4\%) patients were previously untreated, whereas $116(61.1 \%)$ had previously received and/or were receiving treatment for CLL (missing information: 1 patient). Number of lines of treatment were: 1 in 62,2 in 30,3 in 15,4 in $6,>4$ in 3 patients. Sixty-five patients $(34.2 \%)$ were receiving treatment for CLL at the time of COVID-19 diagnosis: 44 were on a BTK inhibitor (ibrutinib 39, acalabrutinib 4, zanubrutinib 1), 9 on venetoclax-based regimens, 3 on idelalisib, 3 
Table 1 Patients' characteristics.

\begin{tabular}{|c|c|}
\hline Characteristic & Result \\
\hline Median age, years (range, years) & $72(48-94)$ \\
\hline Male/female & $126 / 64$ \\
\hline \multicolumn{2}{|l|}{ Diagnosis } \\
\hline CLLIMBL (\%) & $183 / 2(96)$ \\
\hline $\operatorname{SLL}(\%)$ & $5(3)$ \\
\hline $\begin{array}{l}\text { Patients with comorbidities at the time of } \\
\text { COVID-19 }(\%)\end{array}$ & $145(76)$ \\
\hline Median number of comorbidities (range) & $2(1-9)$ \\
\hline $\begin{array}{l}\text { Median time between CLL diagnosis and } \\
\text { COVID-19, months (range, months) }\end{array}$ & $81(0-30$ years $)$ \\
\hline \multicolumn{2}{|l|}{ Type of comorbidities } \\
\hline Hypertension (\%) & $78(54)$ \\
\hline Diabetes mellitus (\%) & $35(24)$ \\
\hline COPD $(\%)$ & $9(6)$ \\
\hline Cardiovascular (\%) & $42(29)$ \\
\hline Respiratory $(\%)$ & $21(14)$ \\
\hline Other malignancies $(\%)$ & $18(12)$ \\
\hline \multicolumn{2}{|l|}{ CLL treatment history } \\
\hline Previously untreated (\%) & $73(38.6)$ \\
\hline Received treatment $(\%)$ & $116(61.4)$ \\
\hline 1 line of treatment & 62 \\
\hline 2 lines of treatment & 30 \\
\hline 3 lines of treatment & 15 \\
\hline 4 lines of treatment & 6 \\
\hline$>4$ lines of treatment & 3 \\
\hline Missing information & 1 \\
\hline On treatment at the time of COVID-19 (\%) & $65(34.2 \%)$ \\
\hline $\begin{array}{l}\text { BTK inhibitor (acalabrutinib, ibrutinib, } \\
\text { zanubrutinib) (\%) }\end{array}$ & $44(67.7)$ \\
\hline Venetoclax-based regimens $(\%)$ & $9(13.8)$ \\
\hline Idelalisib (\%) & $3(4.6)$ \\
\hline Chlorambucil \pm obinutuzumab $(\%)$ & $3(4.6)$ \\
\hline Bendamustine + rituximab $(\%)$ & $2(3.1)$ \\
\hline Other (chemotherapy \pm steroids) $(\%)$ & $4(6.2 \%)$ \\
\hline \multicolumn{2}{|l|}{ Hypogammaglobulinemia } \\
\hline Present $(\%)$ & $89(57.8)$ \\
\hline Absent $(\%)$ & $65(42.2)$ \\
\hline Missing information & 36 \\
\hline
\end{tabular}

$C L L$ chronic lymphocytic leukemia, SLL small lymphocytic lymphoma, $M B L$ monoclonal B-cell lymphocytosis, $C O P D$ chronic obstructive pulmonary disease, BTK Bruton tyrosine kinase.

on chlorambucil \pm obinutuzumab, 2 on bendamustine + rituximab (BR), and 4 on other regimens (steroids and/or chemotherapy, mainly for autoimmune complications). Another 51 patients $(26.8 \%)$ had been previously treated but were not receiving treatment at the time of COVID-19 diagnosis. Amongst these, $19(37.3 \%)$ had received the following therapies in the previous 12 months: fludarabine, cyclophosphamide, and rituximab (FCR, 5), BR (4), other CITs (4), allotransplant (1), novel agents [ibrutinib (3), venetoclax (1), idelalisib + rituximab (1); no available information regarding treatment cessation]. Thirty-two of 51 patients $(62.7 \%)$ had been treated $>1$ year prior to COVID-19 with the following regimens: FCR (10), BR (3), ibrutinib \pm rituximab (4), venetoclax (1), other CITs (13), experimental agents (1). Overall, the median time between the last line of treatment and COVID-19 was 20 months (range 13 months to 10 years).

Eighty-nine of 154 patients (57.8\%) for whom this information was available had documented hypogammaglobulinemia at the time of COVID-19.

Detailed information about the comorbidity and treatment profiles of the studied patients is given in Supplementary material and Supplementary Table 3.

\section{COVID-19 manifestations and management}

Patients with CLL and COVID-19 presented with fever $(165 / 190,87 \%)$ and respiratory symptoms, including cough $(93 / 190,49 \%)$ and dyspnea $(92 / 190,48 \%)$. Other common manifestations included fatigue $(32 / 190,17 \%)$, diarrhea $(22 / 190,12 \%)$, myalgias/arthralgias (19/190, $10 \%)$, headache $(13 / 190,7 \%)$, while anosmia/ageusia $(5 / 190,3 \%)$, nausea and vomiting $(5 / 190,3 \%)$, and abdominal pain $(3 / 190,2 \%)$ were rare (Table 2).

Twenty-one of 190 patients $(11.1 \%)$ were managed at home, while 169 (88.9\%) required hospitalization, in 112/ 169 with need of oxygen supplementation, 39 in the intensive care unit. Most patients (168/187, information missing only in 3 cases) received at least 1 pharmacologic treatment for COVID-19, including: hydroxychloroquine or similar $(76 \%)$, antivirals $(50 \%)$, azithromycin $(47 \%)$, anti-IL6 and/or anti-IL6R monoclonal antibodies (23\%) (Table 2).

Among 65 patients receiving treatment for CLL at the time of COVID-19, $54(83 \%)$ stopped treatment (acalabrutinib $4 / 4$, ibrutinib 30/39, zanubrutinib $1 / 1$, idelalisib $2 / 3$, venetoclax \pm rituximab 9/9, CIT, and/or steroids 8/9). Among those who continued treatment as planned, nine were on ibrutinib, one on idelalisib and one was receiving steroid treatment due to autoimmune hemolytic anemia. Of 45 patients with severe COVID-19 under CLL-specific treatment, 31 were receiving BTK inhibitors: the infection outcome in these 31 patients was resolution (14), still unresolved (4), death (13). In 13 patients with less severe COVID-19 on BTK inhibitors, the outcome was resolution (7), still unresolved (4) and death (1), with 1 missing.

After a median follow-up of 23 days (range 2-86), 96 patients completely recovered (median time to recovery 22.5 days, range 2-86), 37 were still under medical care and 56 died; infection outcome was missing only in 1 case 
Table 2 Clinical presentation and management of patients with COVID-19.

\begin{tabular}{lc}
\hline Characteristic & Result \\
\hline Symptoms & No (\%) \\
Fever & $165(87)$ \\
Cough & $93(49)$ \\
Dyspnea & $92(48)$ \\
Myalgias/arthralgias & $29(15)$ \\
Nausea and vomiting & $5(3)$ \\
Diarrhea & $22(12)$ \\
Fatigue & $32(17)$ \\
Anosmia/ageusia & $5(3)$ \\
Nasal discharge & $5(3)$ \\
Sore throat & $8(4)$ \\
Abdominal pain & $5(3)$ \\
Management of COVID-19 & \\
Confinement at home & $21(11)$ \\
Hospitalization without need of oxygen & $18(10)$ \\
Hospitalization with need of oxygen & $112(59)$ \\
Intensive care & $39(20)$ \\
Pharmacological treatment for COVID-19 & \\
Antivirals & $95(50)$ \\
Hydroxychloroquine or similar & $145(76)$ \\
Azithromycin & $90(47)$ \\
Monoclonal antibodies against IL6/IL6R & $43(23)$ \\
COVID-19 outcome & \\
Resolution & $96(51)$ \\
Still under medical care & $37(19)$ \\
Death & $56(30)$ \\
Missing & 1 \\
\hline
\end{tabular}

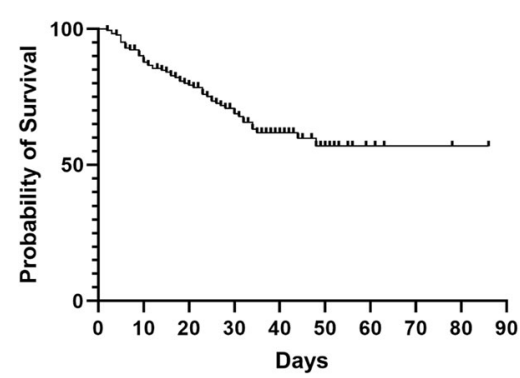

Fig. 1 Overall survival in our patient cohort $(n=190)$. Overall survival of patients with CLL and COVID-19 in days from suspected COVID-19 until last follow-up.

(Table 2). Median OS was not reached, with $69 \%$ of patients alive at 30 days (Fig. 1).

\section{Predictors of outcome of COVID-19 in CLL}

Comparisons between 151 patients who required hospitalization with oxygen support and/or were admitted to the
Table 3 Patients' disposition based on COVID-19 severity.

\begin{tabular}{|c|c|c|c|}
\hline Variable & $\begin{array}{l}\text { Severe } \\
\text { COVID-19 } \\
(n=151)\end{array}$ & $\begin{array}{l}\text { Nonsevere } \\
\text { COVID-19 } \\
(n=39)\end{array}$ & $p$ \\
\hline \multicolumn{4}{|l|}{ Age } \\
\hline$\geq 65$ years $(\%)$ & $112(74.2)$ & $17(43.6)$ & \multirow[t]{2}{*}{$<\mathbf{0 . 0 5}$} \\
\hline$<65$ years $(\%)$ & $39(25.8)$ & $22(56.4)$ & \\
\hline \multicolumn{4}{|l|}{ Gender } \\
\hline Male $(\%)$ & $98(64.9)$ & $28(71.8)$ & \multirow[t]{2}{*}{ n.s. } \\
\hline Female $(\%)$ & $53(35.1)$ & $11(28.2)$ & \\
\hline $\begin{array}{l}\text { Median time between } \\
\text { CLL diagnosis and } \\
\text { COVID-19 }\end{array}$ & 88 & 71 & n.s. \\
\hline \multicolumn{4}{|l|}{ Treatment for CLL } \\
\hline Untreated $(\%)$ & $64(42.7)$ & $9(23.1)$ & \multirow[t]{2}{*}{$<\mathbf{0 . 0 5}$} \\
\hline Treated $(\%)$ & $86(57.3)$ & $30(76.9)$ & \\
\hline \multicolumn{4}{|c|}{ Treatment during the last 12 months or ongoing } \\
\hline Yes $(\%)$ & $60(39.7)$ & $24(61.5)$ & \multirow[t]{2}{*}{$<\mathbf{0 . 0 5}$} \\
\hline $\begin{array}{l}\text { Untreated or treated }> \\
12 \text { months before }(\%)\end{array}$ & $91(60.3)$ & $15(38.5)$ & \\
\hline \multicolumn{4}{|l|}{ Treatment } \\
\hline 1 previous line $(\%)$ & $48(31.8)$ & $14(35.9)$ & \multirow[t]{2}{*}{ n.s. } \\
\hline $\begin{array}{l}2 \text { or more previous } \\
\text { lines }(\%)\end{array}$ & $38(25.2)$ & $16(41.0)$ & \\
\hline \multicolumn{4}{|l|}{ Comorbidities } \\
\hline$>2$ & $46(30.5)$ & $12(30.8)$ & \multirow[t]{2}{*}{ n.s. } \\
\hline$\leq 2$ & $105(69.5)$ & $27(69.2)$ & \\
\hline \multicolumn{4}{|l|}{ Hypogammalobulinemia } \\
\hline Yes & $66(55.0)$ & $23(67.6)$ & \multirow[t]{2}{*}{ n.s. } \\
\hline No & $54(45.0)$ & $11(32.4)$ & \\
\hline \multicolumn{4}{|l|}{ Outcome } \\
\hline Alive $(\%)$ & $96(63.6)$ & $37(97.4)$ & \multirow[t]{2}{*}{$<0.0000$} \\
\hline Dead $(\%)$ & $55(36.4)$ & $1(2.6)$ & \\
\hline
\end{tabular}

Bold values indicate statistical significance $p<0.05$.

intensive care unit, thus experiencing severe COVID-19, versus 39 patients confined at home and/or requiring hospitalization without need of oxygen, therefore, considered to have a less severe infection course, revealed no differences regarding gender, the presence of three or more comorbidities or hypogammaglobulinemia (Table 3).

In contrast, these comparisons revealed significant differences regarding age, CLL treatment history and mortality. In particular, 112/151 (74.2\%) in the severe group were $\geq 65$ years compared to only $17 / 39(43.6 \%)$ in the less severe group $(p<0.05)$. Moreover, significantly $(p<0.05)$ more patients $(91 / 151,60.3 \%)$ in the severe COVID-19 group were off treatment within the last year or had never received treatment for CLL compared to less severe cases (15/39, $38.5 \%$ ). In addition, hospitalization rate due to COVID-19 was significantly different depending on the type of treatment. In particular, 27/1729 (1.6\%) on ibrutinib were 
Table 4 Characteristics of CLL patients who died of COVID-19 $(n=56)$.

\begin{tabular}{|c|c|}
\hline Characteristic & Result \\
\hline Median age, years (range, years) & $75.5(51-91$ \\
\hline Male/female & $38 / 18$ \\
\hline \multicolumn{2}{|l|}{ Diagnosis } \\
\hline CLL $(\%)$ & $54(96.4)$ \\
\hline $\operatorname{SLL}(\%)$ & $1(1.8)$ \\
\hline MBL $(\%)$ & $1(1.8)$ \\
\hline $\begin{array}{l}\text { Patients with comorbidities at the time of COVID- } \\
19(\%)\end{array}$ & $46(82.1)$ \\
\hline Median number of comorbidities (range) & $2(1-9)$ \\
\hline \multicolumn{2}{|l|}{ Type of comorbidities } \\
\hline Hypertension (\%) & $26(46.4)$ \\
\hline Diabetes mellitus $(\%)$ & $10(17.9)$ \\
\hline $\operatorname{COPD}(\%)$ & $4(7.1)$ \\
\hline Cardiovascular (\%) & $10(17.9)$ \\
\hline Respiratory (\%) & $6(10.7)$ \\
\hline Other malignancies $(\%)$ & $4(7.1)$ \\
\hline \multicolumn{2}{|l|}{ CLL treatment history } \\
\hline Previously untreated (\%) & $18(32)$ \\
\hline Received treatment $(\%)$ & $38(68)$ \\
\hline 1 line of treatment & 21 \\
\hline 2 lines of treatment & 8 \\
\hline 3 lines of treatment & 5 \\
\hline 4 lines of treatment & 3 \\
\hline$>4$ lines of treatment & 1 \\
\hline On treatment at the time of COVID-19 (\%) & $19(34)$ \\
\hline BTK inhibitor (acalabrutinib, ibrutinib) & 12 \\
\hline Venetoclax-based regimens & 1 \\
\hline Idelalisib & 1 \\
\hline Chemoimmunotherapy & 3 \\
\hline Corticosteroids & 1 \\
\hline Other (chemotherapy \pm steroids) & 1 \\
\hline \multicolumn{2}{|l|}{ Hypogammaglobulinemia } \\
\hline Present $(\%)$ & $32(64)$ \\
\hline Absent $(\%)$ & $18(36)$ \\
\hline Missing information & 6 \\
\hline \multicolumn{2}{|l|}{ Pharmacological treatment for COVID-19 } \\
\hline Antivirals $(\%)$ & $30(54)$ \\
\hline Hydroxychloroquine or similar (\%) & $38(68)$ \\
\hline Azithromycin $(\%)$ & $18(32)$ \\
\hline Monoclonal antibodies against IL6/IL6R (\%) & $9(16)$ \\
\hline \multicolumn{2}{|l|}{ Management } \\
\hline Confinement at home & $1(1.8)$ \\
\hline Hospitalization without need of oxygen & 0 \\
\hline Hospitalization with need of oxygen & $39(69.6)$ \\
\hline Intensive care & $16(28.6)$ \\
\hline
\end{tabular}

$C L L$ chronic lymphocytic leukemia, $S L L$ small lymphocytic lymphoma, $M B L$ monoclonal B-cell lymphocytosis, COPD chronic obstructive pulmonary disease, $B T K$ Bruton tyrosine kinase. hospitalized because of COVID-19, 8/442 (1.8\%) on venetoclax and $18 / 428(4.2 \%)$ on CIT with a higher risk in those treated with CIT compared to ibrutinib (odds ratio (OR) $2.77,95 \%$ CI 1.51-5.10, $p<0.01$ ) and venetoclax (OR 2.77, 95\% CI 1.02-5.54, $p<0.05$ ). Moreover, hospitalization rate for severe COVID-19 was lower for those on ibrutinib (OR 0.44 [95\% CI 0.20-0.96] $p<0.05$ ) as compared to those receiving any other CLL-specific treatment or off treatment.

The disposition of patients who died because of COVID19 is reported in Table 4. Unsurprisingly, the death rate was significantly $(p<0.00001)$ higher in severe $(55 / 151,36.4 \%)$ versus less severe COVID-19 cases (1/38 cases with available data; $2.6 \%$ ). Of note, however, amongst those who died because of severe COVID-19, age was not found to associate with mortality risk $[16 / 61$ patients $(26.2 \%)<65$ years died versus $40 / 129$ (31\%) patients $\geq 65$ years; $p=$ non significant].

\section{Discussion}

To the best of our knowledge, we present here the largest European series of patients with CLL infected by SARSCoV-2 and experiencing COVID-19. Among the European cases (96.8\% of the total) included in this project, almost $90 \%$ originate from Italy and Spain, hence mirroring the dynamics of the SARS-CoV-2 pandemic in Europe with Italy being the first country in number of infected individuals followed by Spain, with a lower incidence, e.g., in Greece or Northern countries.

Patients were strictly selected and included in this retrospective analysis only if they had a confirmed COVID-19 diagnosis by molecular testing and were followed at the participating sites. In order to avoid the possibility of ascertainment biases due to the fact that in most countries only patients with relevant symptoms are tested for SARSCoV-2, we tried to draw conclusions in particular from cases requiring hospitalization with or without oxygen support and/or intensive care admission, making it more comparable in all different national situations.

In line with previous reports in the general population, also in our cohort older age was associated with more severe COVID-19 manifestations: in particular, the group of patients admitted to the hospital requiring oxygen and/or ventilatory support was enriched for patients $\geq 65$ years compared to those with milder disease. The presence of three or more comorbidities was not significantly different in patients hospitalized with severe versus nonsevere disease; moreover, the presence of hypogammaglobulinemia, a frequent laboratory finding in CLL, did not show a relevant impact on the clinical course of COVID-19 patients, probably underscoring the relevance of the inflammatory 
reaction rather than the viral replication (and the capacity to clear it by antibody-mediated immune response) in shaping the severity of the disease.

The potential impact of CLL-specific treatments on the course of COVID-19 still needs to be fully elucidated, with international guidelines suggesting careful evaluation of pros/cons of treatment interruption, in particular in patients on targeted agents [20]. Taking into consideration the potential long-term effect on the immune system and the risk of infections (up to 12 months) particularly for patients treated with CIT, we grouped together patients who were on ongoing or had received recent $(<12$ months) therapies vs patients with no treatments in their lifetime or in the past previous year. Relevant to mention in this respect, our cohort of COVID-19 patients is enriched for patients receiving treatment for CLL compared to a standard population (indeed 2679/15,083, 17.8\% were on treatment at participating sites, while 65/190, 34.2\% COVID-19 cases were treated). Interestingly, patients under ibrutinib less likely needed hospitalization suggesting a diverse effect of the antileukemic treatments, probably due to a different impact on the immune deficiency and/or dysregulation. Along the same direction, we here report that patients on recent or ongoing treatment were more likely to have a milder COVID-19 compared to those with severe disease who in the vast majority were on a watch and wait followup. It is important to note that a relevant proportion of patients included into the study were on BTK inhibitors but, due to limited numbers of patients treated with other therapies, our study does not allow reaching firm conclusions on potential differences between the different types of treatment (BTK inhibitors vs venetoclax-based regimens vs CIT and/or steroids). Altogether, these data seem to further underscore the possible protective role of specific targeted therapies against a dismal evolution of the SARS-CoV-2 infection $[17,21]$.

Considering the median age of 72 years at the time of COVID-19 occurrence and the presence of at least one comorbidity in $\sim 75 \%$ of our patients (median number of comorbidities 2$)$, the high mortality rate $(55 / 169,32.5 \%)$ among the hospitalized patients reported in this cohort comes as no surprise, though it is apparently higher than expected in the general population (e.g., 13.4\% amongst the general population in Italy) (https://www.epicentro.iss.it/ coronavirus/). However, if we consider only patients with CLL $\geq 70$ years of age, the percentage $(36 / 105,34.3 \%)$ is not considerably different from that of normal individuals $>70$ years (25.5\%, https://www.epicentro.iss.it/coronavirus/) [18]. The difference in the overall mortality rate was seen in all age groups, with young patients ( $<65$ years) succumbing to the disease at rates similar to those of the elderly $(26.2 \%$ and $31.0 \%$, respectively), though the elderly patients were more likely to experience severe COVID-19, as reported above. This evidence is pointing to the fact that when severe COVID-19 occurs, it is probably the underlying leukemic disease with the typical immune dysregulation that predisposes to a dismal outcome, levelling off the death risk and overcoming the effect of age and other comorbidities.

Along the same line of reasoning, the apparent higher rate of hospitalization in our cohort $(141 / 15,083,0.9 \%)$ compared to a much lower rate for example in the Italian population $(0.24 \%) \quad$ (https://www.epicentro.iss.it/corona virus/) may simply be due to older age and higher comorbidity burden of the patients with CLL here reported.

Our study has some limitations: (1) the large majority of CLL cases was provided by only two countries (Italy and Spain), making the case series possibly not fully representative of the COVID-19 course in other countries; (2) cases with more severe clinical presentations had a higher probability of being included in the study due to having a confirmed COVID-19 diagnosis, making it difficult to define the prevalence, morbidity and mortality compared to the general population, that indeed was the aim of our study. To mitigate at least in part these limitations, we restricted our analyses to hospitalized patients that are more objectively recorded in all different national situations. Despite these limitations, our series thoroughly describes COVID19 outcome in a large cohort of patients with CLL in Europe and suggests that these patients may be at a higher risk of developing symptomatic COVID-19 and of succumbing to the disease due to the underlying leukemia, while at least some CLL treatments may favorably influence COVID-19 course. This finding warrants further investigation to understand the potential protective role of CLL treatment, in particular with BTK inhibitors, on the clinical course, morbidity and mortality of COVID-19 patients with CLL.

Acknowledgements This work was in part supported by Associazione Italiana per la Ricerca sul Cancro-AIRC, Milano, Italy (Investigator Grant \#20246 and Special Program on Metastatic Disease-5 per mille \#21198); ERA NET TRANSCAN-2 Joint Transnational Call for Proposals: JTC 2014 (project \#143 GCH-CLL) and JTC 2016 (project \#179 NOVEL), project code (MIS) 5041673; Bando della Ricerca Finalizzata 2018, Ministero della Salute, Roma, Italy (progetto RF-2018-12368231); the SARS-CoV-2 research mission of the Greek Secretariat for Research and Technology; BEAT Leukemia and AIL-FE.

\section{Compliance with ethical standards}

Conflict of interest LS received honoraria from AbbVie, AstraZeneca, Gilead, Janssen. GMR received honoraria from Abbvie, Gliead, and Janssen and research funding from Gilead. MaMo received onoraria from Janssen and Roche. CV received consultancy fees from Janssen, outside the submitted work. JAG received honoraria from AbbVie, AstraZeneca, Gilead, Janssen, and Roche. Research funding from AbbVie, Gilead, and Janssen. JAH received honoraria for lectures and Advisory Boards from Janssen, Abbvie, Roche, Gilead, AstraZeneca. 
CUN received research support and/or honoraria from Abbvie, AstraZeneca, CSL Behring, Janssen, and Sunesis. GR received honoraria from AbbVie, Gilead, Janssen. EG received travel grants, honoraria as consultant and/or speaker bureau from Janssen, Abbvie, Roche, and Gilead. MoMo received consultant fees from Gilead. MV received honoraria for Advisory boards from Janssen and Roche. SI received Honoraria Janssen and Gilead. AJ received travel grants, speaker fees or consultancies: Amgen, Abbvie, Celgene, Janssen, Gilead, Novartis, Sanofi-Genzyme, Roche. AF received honoraria from AbbVie. MaSp received honoraria from AbbVie, Gilead, and Janssen. ML received honoraria for Advisory board and travel compensation from Janssen, Abbvie, and Roche. EVDS received compensation for teaching activities from Amgen. EV received travel grants from Abbvie, Gilead, Jannsenns, and Roche, research grants from Abbvie, Gilead, and Roche. LF received honoraria from AbbVie, Janssen (Advisory role or Lecturer). MF received honoraria from Abbvie, Janssen, Gilead. YH received honoraria from AbbVie, AstraZeneca, Janssen, Medison, Sanofi, and Roche. OJ received Honoraria from Abbvie, Janssen, and Roche. APK received research support: Abbvie, Janssen, Roche/Genentech, AstraZeneca; Adboard: Abbvie, Janssen, Roche/Genentech, AstraZeneca; speakersfee: Janssen, AstraZeneca, Abbvie. SK received Travel grant from Celgene, research funding from Janssen, Abbvie. MA received travel support and advisory boards from AbbVie, Janssen-Cilag, Celgene, Novartis. DR received honoraria from Abbvie, AstraZeneca, Gilead, Janssen, Loxo, and Verastem, and research grants from Abbvie, AstraZeneca, Cellestia, Gilead, Janssen. NS received consulting fees from AbbVie, Roche, Janssen. CT received honorarium and research funding from Janssen, Beigene, and Abbvie. MC received research funding from Janssen and Karyopharm Therapeutics, and personal fees from Janssen, Gilead, Abbvie, and Shire, outside the submitted work. KS received honoraria from Abbvie, Acerta/AstraZeneca, Gilead, Janssen, and research funding from Abbvie, Gilead, Janssen. AC received honoraria from AbbVie, AstraZeneca, Gilead, Janssen. RF received honoraria from Abbvie, Gliead, Janssen, AstraZeneca, Amgen, Incyte, Novartis. PG received honoraria from AbbVie, Adaptive, Acerta/ AstraZeneca, ArQule, BioGene, Dynamo, Gilead, Janssen, MEI, Sunesis, and Research funding from AbbVie, Gilead, Janssen, Sunesis. TC, GQ, FM, MB, JM, TM, FMQ, JD, RM, CC, GI, LDP, LL, EL, FRM, MaSi, LT, EW, RR, DB, MRDP, GDP, MG, TH, LM, LO VMP, PS, MY, BP, GB, AC, GdS, EN, MaDi, MiDo, AE, YH, OK, MOP, MP, AS, OA, NS, VS, MZ, GR, AR, EM have no conflict of interest to disclose.

Publisher's note Springer Nature remains neutral with regard to jurisdictional claims in published maps and institutional affiliations.

\section{References}

1. Zhu N, Zhang D, Wang W, Li X, Yang B, Song J, et al. A novel coronavirus from patients with pneumonia in China, 2019. N Engl J Med. 2020;382:727-33.

2. Huang C, Wang Y, Li X, Ren L, Zhao J, Hu Y, et al. Clinical features of patients infected with 2019 novel coronavirus in Wuhan, China. Lancet 2020;395:497-506.

3. Zhao R, Li M, Song H, Chen J, Ren W, Feng Y, et al. Early detection of SARS-CoV-2 antibodies in COVID-19 patients as a serologic marker of infection. Clin Infect Dis. 2020:ciaa523. https://doi.org/10.1093/cid/ciaa523.

4. Chen G, Wu D, Guo W, Cao Y, Huang D, Wang H, et al. Clinical and immunological features of severe and moderate coronavirus disease 2019. J Clin Investig. 2020;130:2620-9.
5. England JT, Abdulla A, Biggs CM, Lee AYY, Hay KA, Hoiland $\mathrm{RL}$, et al. Weathering the COVID-19 storm: lessons from hematologic cytokine syndromes. Blood Rev. 2020:100707. https://doi. org/10.1016/j.blre.2020.100707.

6. Liang WH, Guan WJ, Li CC, Li YM, Liang HR, Zhao Y, et al. Clinical characteristics and outcomes of hospitalised patients with COVID-19 treated in Hubei (epicenter) and outside Hubei (nonepicenter): a nationwide analysis of China. Eur Respir J. 2020; 55:2000562.

7. He W, Chen L, Chen L, Yuan G, Fang Y, Chen W, et al. COVID-19 in persons with haematological cancers. Leukemia. 2020;34:1637-45.

8. Yang K, Sheng Y, Huang C, Jin Y, Xiong N, Jiang K, et al. Clinical characteristics, outcomes, and risk factors for mortality in patients with cancer and COVID-19 in Hubei, China: a multicentre, retrospective, cohort study. Lancet Oncol. 2020;21:904-13.

9. Dai M, Liu D, Liu M, Zhou F, Li G, Chen Z, et al. Patients with cancer appear more vulnerable to SARS-CoV-2: a multicenter study during the COVID-19 outbreak. Cancer Discov. 2020;10: 783-91.

10. Tian J, Yuan X, Xiao J, Zhong Q, Yang C, Liu B, et al. Clinical characteristics and risk factors associated with COVID-19 disease severity in patients with cancer in Wuhan, China: a multicentre, retrospective, cohort study. Lancet Oncol. 2020;21: 893-903.

11. Martín-Moro F, Marquet J, Piris M, Michael BM, Saez AJ, Corona M, et al. Survival study of hospitalised patients with concurrent COVID-19 and haematological malignancies. Br J Haematol. 2020; 190:e16-20.

12. Baumann T, Delgado J, Montserrat E. CLL and COVID-19 at the Hospital Clinic of Barcelona: an interim report. Leukemia. 2020;34:1954-6.

13. Jin XH, Zheng KI, Pan KH, Xie YP, Zheng MH. COVID-19 in a patient with chronic lymphocytic leukaemia. Lancet Haematol. 2020;7:e351-e2.

14. Forconi F, Moss P. Perturbation of the normal immune system in patients with CLL. Blood. 2015;126:573-81.

15. Riches JC, Davies JK, McClanahan F, Fatah R, Iqbal S, Agrawal S, et al. T cells from CLL patients exhibit features of T-cell exhaustion but retain capacity for cytokine production. Blood. 2013;121: 1612-21.

16. Maffei R, Maccaferri M, Arletti L, Fiorcari S, Benatti S, Potenza $\mathrm{L}$, et al. Immunomodulatory effect of ibrutinib: reducing the barrier against fungal infections. Blood Rev. 2020;40:100635.

17. Treon SP, Castillo JJ, Skarbnik AP, Soumerai JD, Gobrial IM, Guerrera ML, et al. The BTK inhibitor ibrutinib may protect again pulmonary injury in COVID-19-infected patients. Blood. 2020;135:1912-5.

18. Cuneo A, Scarfo L, Reda G, Varettoni M, Quaglia FM, Marchetti $\mathrm{M}$, et al. Chronic lymphocytic leukemia management in italy during the COVID-19 Pandemic. A campus CLL report. Blood. 2020:2020006854. https://doi.org/10.1182/blood.2020006854.

19. Hallek M, Cheson BD, Catovsky D, Caligaris-Cappio F, Dighiero $\mathrm{G}$, Dohner $\mathrm{H}$, et al. iwCLL guidelines for diagnosis, indications for treatment, response assessment, and supportive management of CLL. Blood. 2018;131:2745-60.

20. Rossi D, Shadman M, Condoluci A, Brown JR, Byrd JC, Gaidano G, et al. How we manage patients with chronic lymphocytic leukemia during the SARS-CoV-2 Pandemic. HemaSphere. 2020;4:e432. https://doi.org/10.1097/HS9.0000000000000432

21. Roschewski M, Lionakis MS, Sharman JP, Roswarski J, Goy A, Monticelli MA, et al. Inhibition of Bruton tyrosine kinase in patients with severe COVID-19. Sci Immunol. 2020;5: eabd0110. 


\section{Affiliations}

Lydia Scarfò $^{1} \cdot$ Thomas Chatzikonstantinou $^{2} \cdot$ Gian Matteo Rigolin $^{3} \cdot$ Giulia Quaresmini $^{4} \cdot$ Marina Motta $^{5}$. Candida Vitale $^{6}$ - Jose Antonio Garcia-Marco $\mathbb{D}^{7} \cdot$ José Ángel Hernández-Rivas $\mathbb{D}^{8} \cdot$ Fatima Mirás $^{9} \cdot$ Mónica Baile $^{10}$.

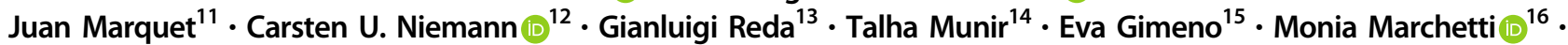
Francesca Maria Quaglia ${ }^{17}$. Marzia Varettoni ${ }^{18}$. Julio Delgado ${ }^{19}$. Sunil lyengar ${ }^{20}$ - Ann Janssens ${ }^{21} \cdot$ Roberto Marasca $^{22}$. Angela Ferrari ${ }^{23}$. Carolina Cuéllar-García $\circledast^{24}$. Gilad Itchaki ${ }^{25}$ - Martin Špaček ${ }^{26}$ • Lorenzo De Paoli ${ }^{27} \cdot$ Luca Laurenti $^{28}$. Mark-David Levin ${ }^{29}$. Enrico Lista ${ }^{30}$. Francesca R. Mauro ${ }^{31}$ - Martin Šimkovič ${ }^{32}$ • Ellen Van Der Spek ${ }^{33}$.

Elisabeth Vandenberghe ${ }^{34} \cdot$ Livio Trentin ${ }^{35}$. Ewa Wasik-Szczepanek ${ }^{36} \cdot$ Rosa Ruchlemer $^{37}$ - Dominique Bron ${ }^{38}$. Maria Rosaria De Paolis ${ }^{39}$. Giovanni Del Poeta ${ }^{40}$ - Lucia Farina ${ }^{41}$. Myriam Foglietta ${ }^{42}$ - Massimo Gentile ${ }^{43}$. Yair Herishanu ${ }^{44} \cdot{\text { Tobias Herold } \mathbb{D}^{45} \cdot \text { Ozren Jaksic }^{46} \text { - Arnon P. Kater }}^{47} \cdot$ Sabina Kersting ${ }^{48} \cdot$ Lara Malerba $^{49}$. Lorella Orsucci ${ }^{50}$ - Viola Maria Popov $\mathbb{D}^{51} \cdot$ Paolo Sportoletti ${ }^{52} \cdot$ Mohamed Yassin ${ }^{53}$. Barbara Pocali ${ }^{54} \cdot$ Gabor Barna $^{55}$. Annalisa Chiarenza ${ }^{56}$. Gimena dos Santos $\mathbb{D}^{57}$. Eugene Nikitin ${ }^{58} \cdot$ Martin Andres $\mathbb{1}^{59} \cdot$ Maria Dimou $\mathbb{1}^{60}$. Michael Doubek $\mathbb{1}^{61}$ - Alicia Enrico ${ }^{62} \cdot$ Yervand Hakobyan ${ }^{63}$. Olga Kalashnikova ${ }^{64}$ - Macarena Ortiz Pareja ${ }^{65}$. Maria Papaioannou ${ }^{66}$ - Davide Rossi ${ }^{67,68}$ - Nimish Shah ${ }^{69} \cdot$ Amit Shrestha $^{70}$. Oana Stanca ${ }^{71} \cdot$ Niki Stavroyianni $^{2}$.

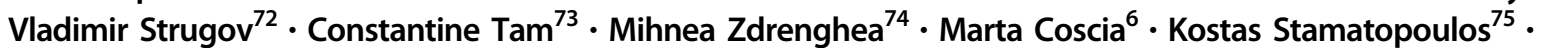
Giuseppe Rossi ${ }^{5} \cdot$ Alessandro Rambaldi $\mathbb{C}^{4} \cdot$ Emili' Montserrat $^{19} \cdot{\text { Robin Foà } \mathbb{C}^{31} \cdot \text { Antonio Cuneo }}^{3} \cdot$ Paolo Ghia $\mathbb{D}^{1}$

1 Università Vita-Salute San Raffaele and IRCC Ospedale San Raffaele, Milan, Italy

2 Hematology Department and HCT Unit, G. Papanicolaou Hospital, Thessaloniki, Greece

3 Hematology Section, Department of Medical Sciences, University of Ferrara, Ferrara, Italy

4 USC Ematologia, ASST PAPA GIOVANNI XXIII, Bergamo, Italy

5 S.C. Ematologia, ASST Spedali Civili, Brescia, Italy

6 University Division of Hematology, A.O.U. Città della Salute e della Scienza di Torino and Department of Molecular Biotechnology and Health Sciences, University of Torino, Torino, Italy

7 Hematology Department, Hospital Universitario Puerta de HierroMajadahonda, Madrid, Spain

8 Hospital Universitario Infanta Leonor, Madrid Universidad Complutense, Madrid, Spain

9 Hospital Universitario 12 de Octubre, Madrid, Spain

10 Servicio de Hematología del Complejo Asistencial Universitario de Salamanca-IBSAL, Salamanca, Spain

11 Hematology Department, Hospital Universitario Ramón y Cajal, Madrid, Spain

12 Department of Hematology, Rigshospitalet, Copenhagen University Hospital, Copenhagen, Denmark

13 U.O.C. Ematologia, Fondazione IRCCS Ca'Granda Ospedale Maggiore Policlinico, Milan, Italy

14 St James's University Hospital, Leeds, UK

15 Hematology Department Hospital del Mar, Barcelona, Spain

16 Hematology Dpt, Az Osp SS Antonio e Biagio e Cesare Arrigo, Alessandria, Italy
17 Department of Medicine, Section of Hematology, University of Verona, Verona, Italy

18 Division of Haematology, Fondazione IRCCS Policlinico San Matteo, Pavia, Italy

19 Dept. of Hematology, Hospital Clinic, IDIBAPS, Barcelona, Spain

20 Department of Haemato-Oncology, Royal Marsden Hospital, London, UK

21 Universitaire Ziekenhuizen Leuven, Leuven, Belgium

22 Hematology Unit, Department of Medical and Surgical Sciences, University of Modena and Reggio Emilia, Modena, Italy

23 Hematology, Azienda USL-IRCCS di Reggio Emilia, Reggio Emilia, Italy

24 Hematology Unit, Consorci Sanitari Terrassa Hospital, Terrassa, Spain

25 Rabin Medical Center, Beilinson Hospital; Sackler Faculty of Medicine, Tel Aviv University, Tel Aviv, Israel

26 1st Department of Medicine-Department of Hematology, First Faculty of Medicine, Charles University and General Hospital in Prague, Prague, Czech Republic

27 Division of Hematology, Department of Translational Medicine, University of Eastern Piedmont, Novara, Italy

28 Dipartimento Scienze Radiologiche ed Ematologiche, Divisione di Ematologia Fondazione Policlinico universitario A Gemelli, Roma, Italy

29 Department of Internal Medicine, Albert Schweitzer Hospital, Dordrecht, The Netherlands

30 Struttura Semplice Dipartimentale di Ematologia, Presidio Ospedaliero Santa Chiara, Trento, Italy 
31 Hematology, Department of Translational and Precision Medicine, Sapienza University, Policlinico Umberto I, Rome, Italy

32 Department of Internal Medicine-Haematology, University Hospital and Medical School Hradec Kralove, Hradec Kralove, Czech Republic Medical University in Lublin, Lublin, Poland

Department of Hematology, Shaare Zedek Medical Center, Jerusalem, Israel

\section{dei Tumori, Milano, Italy}

42 SC Ematologia, AO S. Croce e Carle, Cuneo, Italy

43 UOC Ematologia AO Cosenza, Cosenza, Italy

44 Department of Hematology, Tel Aviv Sourasky Medical Center, and Sackler Faculty of Medicine, Tel Aviv University, TelAviv, Israel

Department of Medicine III, University Hospital, LMU Munich, Munich, Germany

Department of Hematology, University hospital Dubrava, Zagreb, Croatia

47 Dep of Hematology, Cancer Center Amsterdam, Amsterdam University Medical Centers, Amsterdam, The Netherlands

Department of Hematology, Haga Hospital, The Hague, The Netherlands

Hematology Unit, Azienda Ospedaliera "Ospedali Riuniti Marche Nord", Pesaro, Italy

50 Division of Hematology II, San Giovanni Battista Hospital, Torino, Italy

51 Colentina Clinical Hospital Bucharest, Bucharest, Romania

52 Institute of Hematology and Center for Hemato-Oncological Research, Ospedale S. Maria della Misericordia, University of Perugia, Perugia, Italy

53 National Center for Cancer Care and Research Doha, Doha, Qatar

54 Division of Hematology and Stem Cell Transplantation Unit, Cardarelli Hospital, Naples, Italy
55 1st Department of Pathology and Experimental Cancer Research, Semmelweis University, Budapest, Hungary

56 Division of Hematology, Policlinico, Department of Surgery and Medical Specialties, University of Catania, Catania, Italy

57 Hospital de Clinicas, Montevideo, Uruguay

58 Department of Hematology, Oncology, and Chemotherapy, S. P. Botkin's City Hospital, Moscow, Russia

59 Department of Hematology and Central Hematology Laboratory, Inselspital, Bern University Hospital, University of Bern, Bern, Switzerland

60 1st First Propedeutic Department of Internal Medicine, Hematology Unit, Laikon General Hospital, National and Kapodistrian University of Athens, Medical School of Athens, Athens, Greece

61 University Hospital and CEITEC MU Brno, Brno, Czech Republic

62 Hematology Area, Hospital Italiano La Plata, La Plata, Argentina

63 Hematology Center after Prof. Yeolyan MH RA, Yerevan, Armenia

64 First State Pavlov Medical University of Saint-Petersburg, Raisa Gorbacheva Memorial Institute for Children Oncology, Hematology and Transplantation Russia, Saint-Petersburg, Russia

65 Carlos Haya, Málaga, Spain

66 Hematology Unit, 1st Dept of Internal Medicine, AUTH, AHEPA Hospital, Thessaloniki, Greece

67 Laboratory of Experimental Hematology, Institute of Oncology Research, Bellinzona, Switzerland

68 Division of Hematology, Oncology Institute of Southern Switzerland, Bellinzona, Switzerland

69 Norfolk \& Norwich University NHS Foundation Trust, Norwich, UK

70 Hematology Unit, Nepal Cancer Hospital \& Research Centre, Lalitpur, Nepal

71 "Carol Davila" University of Medicine and Pharmacy, Hematology Clinic, Coltea Clinical Hospital, Bucharest, Romania

72 Hematology Almazov National Medical Research Centre, St. Petersburg, Russia

73 Peter MacCallum Cancer Centre and University of Melbourne, Victoria, Australia

74 Department of Hematology, Iuliu Hatieganu University of Medicine and Pharmacy and Ion Chiricuta Oncology Institute, Cluj-Napoca, Romania

75 Institute of Applied Biosciences, Centre for Research and Technology Hellas, Thessaloniki, Greece 